\title{
Mario Bunge
}

Philosophy Faculty, McGill University, Montréal

(1986)

\section{"Considérations d'un philosophe sur l'économique du néo-conservatisme (néo-libéralisme)"}

\author{
Un document produit en version numérique par Jean-Marie Tremblay, bénévole, \\ professeur de sociologie au Cégep de Chicoutimi \\ Courriel: jmt_sociologue@videotron.ca \\ Site web: http://pages.infinit.net/sociojmt
}

Dans le cadre de la collection: "Les classiques des sciences sociales"

Site web: $\underline{\text { http://www.uqac.uquebec.ca/zone30/Classiques_des_sciences_sociales/index.html }}$

Une collection développée en collaboration avec la Bibliothèque

Paul-Émile-Boulet de l'Université du Québec à Chicoutimi

Site web: http://bibliotheque.uqac.uquebec.ca/index.htm 
Cette édition électronique a été réalisée par Jean-Marie Tremblay, bénévole, professeur de sociologie au Cégep de Chicoutimi à partir de :

Monsieur Mario Bunge, professeur de philosophie, McGill University, Montréal,

“ Considérations d'un philosophe sur l'économique du néo-conservatisme (néolibéralisme)*" (1986)

Une édition électronique réalisée à partir de l'article de M. Mario Bunge, philosophe et professeur de philosophie [Faculté de philosophie, McGill University], "Considérations d'un philosophe sur l'économique du néo-conservatisme (néolibéralisme)*" in ouvrage sous la direction de Lizette Jalbert et Laurent Lepage, Néoconservatisme et restructuration de l'État. Canada - États-Unis - Angleterre. Première partie : Lectures du néo-conservatisme (pp. 49 à 70) Collection Études d'économie politique. Montréal : Les Presses de l'Université du Québec, 1986, 274 pp.

(*) L'essentiel de ce texte est tiré d'une étude inédite, intitulée The Methodological Crisis of Economics. Une première version de cette étude a été publiée en espagnol, sous le titre Economia y filosofia, Madrid, Tecnos, 1982, 1985.

Autorisation accordée par le professeur Bunge le 30 décembre 2003.

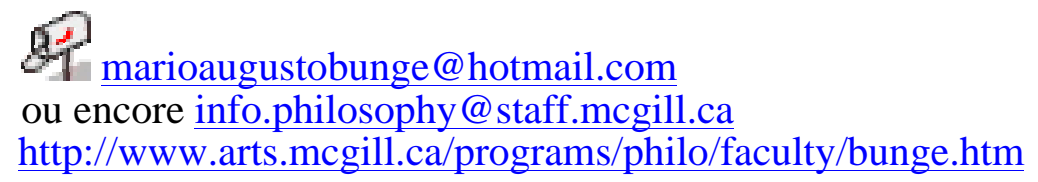

Polices de caractères utilisée :

Pour le texte: Times, 12 points.

Pour les citations : Times 10 points.

Pour les notes de bas de page : Times, 10 points.

Édition électronique réalisée avec le traitement de textes Microsoft Word 2001 pour Macintosh.

Mise en page sur papier format

LETTRE (US letter), 8.5' x 11')

Édition complétée le 31 décembre 2003 à Chicoutimi, Québec. 


\section{Table des matières}

Introduction

1. Fondements théoriques

2. La politique économique

Conclusion 


\section{“Considérations d'un philosophe sur l'économique du néo-conservatisme (néo-libéralisme)" 1}

\section{Introduction}

Le néo-conservatisme sévit aujourd'hui dans de nombreux pays, certains développés, d'autres moins. La respectabilité dont il s'honore repose, au premier chef, sur un État de fait purement négatif, soit l'impuissance du libéralisme et du socialisme à assurer une croissance économique continue. Cependant, les principes que met de l'avant cette politique sont au bas mot suspects, son éthique incertaine et son efficacité reste encore à démontrer.

Nous nous limiterons ici à l'étude des fondements théoriques de l'économique proposée par le néo-conservatisme et de la philosophie morale qui la sous-tend. Nous examinerons aussi du point de vue méthodologique les politiques économiques en général et leur rapport avec la théorie, tant économique qu'éthique.

Ce propos devrait être fructueux intellectuellement parlant puisque, de tout temps, la méthodologie en économie est reconnue comme étant un domaine particulièrement

L'essentiel de ce texte est tiré d'une étude inédite, intitulée The Methodological Crisis of Economics. Une première version de cette étude a été publiée en espagnol, sous le titre Economia y filosofia, Madrid, Tecnos, 1982, 1985. Une première version de la traduction de ce texte a été assurée par les services de traduction de l'Université McGill. 
épineux. Ainsi, l'échec d'une politique économique peut être attribuable soit à ses bases théoriques, soit à des lacunes dans sa mise en oeuvre. Autre motif d'intérêt inhérent à l'étude philosophique de la science économique : les prémisses morales n'en sont pas toujours explicites. Ainsi, on s'accorde à reconnaître que le concept marxiste de la plus-value est de nature économico-morale, mais rares sont ceux qui repensent la condamnation de la planification par Hayek ou le plaidoyer de Friedman en faveur de la liberté de choix dans l'optique de principes moraux.

Notre étude a aussi une portée sociale importante car le destin de millions de gens est, en partie du moins, entre les mains de politiciens néo-conservateurs, conseillés par quelques économistes néo-libéraux. Il est impossible de comprendre et d'évaluer leurs décisions si l'on n'en connaît pas les concepts sous-jacents et si l'on ne cherche pas à mesurer leur pertinence tant aux plans empirique que moral.

Mais que peut valoir l'opinion d'un philosophe formé à la physique sur ces questions ? Ne sera-t-il pas porté à faire certaines erreurs ? Sans doute, mais vraisemblablement, si c'est le cas, leur incidence n'aura pas la portée de celles commises par maints lauréats du prix Nobel de science économique. Et quant à ses idées, justes ou erronées, elles risquent peu d'affecter de façon désastreuse le destin de millions de gens, ni de mettre toute une civilisation en péril. De plus, j'oserais avancer que le philosophe pourrait en remontrer à certains économistes sur la nature de la science économique (une semi-science ? une pseudo-science ?), le mode de vérification des théories économiques, les rapports entre les fondements et la mise en application des politiques économiques. Bref, le philosophe pourrait bien être capable de discerner les points faibles de la science économique. Mais, bien sûr, il appartient à l'économiste d'apporter des corrections dans certains cas, d'épurer la conceptualisation dans d'autres et, si la chose est possible, d'en arriver à développer des idées pertinentes et nouvelles, qui pourront transformer l'aride désert de l'économie en un terrain fertile.

\section{Fondements théoriques}

Retour à la table des matières

L'économie néo-conservatrice (ou néo-libérale) est effectivement conservatrice en théorie : il ne s'agit en définitive que d'une version récente de l'économie néo-classique. À l'instar d'autres théories des sciences sociales, l'économie néo-classique repose sur deux ensembles d'hypothèses, les unes implicites, les autres explicites. Considérons d'abord les premières, qu'on pourrait rassembler sous la rubrique : Décalogue psycho-économique néo-classique : 
i. L'individu, le consommateur en particulier, est souverain.

ii. L'homme est insatiable dans ses besoins.

iii. L'homme est essentiellement thésauriseur.

iv. Tous ont des préférences et peuvent les classer logiquement, par ordre de priorité.

v. Tous sont disposés à agir en vue de satisfaire leurs besoins.

vi. L'homme tend à minimiser les efforts à faire pour satisfaire ses besoins : en clair, l'homme déteste travailler.

vii. L'homme est naturellement compétitif et même agressif plutôt que coopératif.

viii. Plus quelqu'un possède d'un bien, moins il en apprécie l'augmentation en quantité (loi de l'utilité marginale décroissante).

ix. L'homme doit constamment faire face à des choix et est ainsi forcé de prendre des décisions.

x. L'homme recherche l'optimisation : il prend les décisions les plus aptes à optimiser son efficacité (ou son bien-être, ou ses gains).

Toutes les théories économiques traitant de l'économie de marché concurrentiel sont censées découler, en dernière analyse, des présupposés contenus dans ce Décalogue individualiste et hédoniste. Et pourtant, rares sont les psychologues qui semblent s'en soucier. Il ne semble pas non plus y avoir eu d'efforts systématiques et intensifs pour en vérifier la justesse autrement que par le critère de la durée : «C'est ancien, donc ça doit être valable ». Ce phénomène peut être attribuable à la croyance selon laquelle les hypothèses formulées ci-dessus parlent d'elles-mêmes (pour ceux qui les ont apprises dans les manuels classiques), ou encore selon un point de vue erroné qui revient à ceci : « on peut dire d'une hypothèse qu'elle est confirmée simplement s'il ne s'est trouvé personne pour l'invalider ». Ce point de vue est énoncé par Machlup dans le sillage de Popper ${ }^{2}$. Mais il va de soi que tout scientifique qui se respecte accorde une importance considérable aux preuves empiriques positives ${ }^{3}$.

Nous convenons volontiers que certaines des hypothèses énumérées ci-dessus semblent établies au-delà de tout doute raisonnable, nommément (v.) et (viii). Mais les autres doivent être remises en question, sinon entièrement, du moins en partie. Par exemple l'individu, loin d'être « souverain » (i.), n'est plutôt, en réalité, que le rouage d'une énorme machine sur laquelle il n'a aucune emprise. Tandis que l'insatiabilité (ii.) semble rare sinon inexistante dans les sociétés pré-industrielles, du moins parmi

F. Machlup, «The Problem of Verification in Economics », Southern Economic Journal, no. 22, 1955 , p. 5.

M. Bunge, Understanding the World, Boston-Dordrecht, Reidel, 1983. 
le menu peuple qui réussit à peine à satisfaire ses besoins les plus criants. La thésaurisation (iii.) est également inconnue dans les sociétés primitives, de sorte qu'il doit s'agir d'un trait acquis et non inné.

Bien que tous les animaux - et non seulement les humains aient des préférences (iv.), les psychologues ont découvert, à la fin des années 1950 et au début des années 1960, que la plupart d'entre nous souffrons souvent d'incohérence et ne sommes pas toujours capables de classer nos préférences par ordre de priorité (strictement ou partiellement). En fait, nous préférons souvent $\mathrm{A}$ à $\mathrm{B}$ et $\mathrm{B}$ à $\mathrm{C}$, mais non $\mathrm{A}$ à $\mathrm{C}$. De plus, les économistes d'obédience néo-classique acceptent la notion de préférence comme allant de soi, plutôt que d'étudier les mécanismes sociaux et psychologiques à l'origine de ces préférences. (Songeons ici à la préférence que manifestent les enfants nord-américains pour le junk food et les boissons gazeuses qui font l'objet de tant de publicité). Pis encore, ils traitent les préférences de la façon la moins réaliste, en prenant comme critère le prix plutôt que le besoin. En fait, une des idées cruciales de la théorie du consommateur est que deux collections de produits composées d'articles de même type, en quantité différente, sont équivalentes pourvu que leur valeur d'échange totale (leur prix) soit la même. Tout consommateur averti devrait donc considérer équivalents un panier contenant neuf pains et un kilo de beurre et un autre contenant un pain et cinq kilos de beurre. Les besoins objectifs ne jouent aucun rôle dans la constitution des courbes d'indifférence, ce qui explique que ces dernières n'aient aucun rapport avec la réalité. Puisque la constitution des fonctions d'utilité repose sur de telles courbes d'indifférence, c'est l'ensemble du corps de l'économique néo-classique qui se trouve à n'avoir avec la réalité qu'un lien des plus ténu.

Il est carrément faux que nous tentions tous de réduire au minimum nos efforts et que nous détestions le travail. L'homme est naturellement porté à l'action et va même jusqu'à s'inventer des occupations lorsqu'il n'a rien à faire. En outre, des expériences ont démontré que les gens, et même les rats, préfèrent travailler à ne rien faire, même si le travail en question est peu satisfaisant, justement parce que l'action porte sa propre récompense ${ }^{4}$. Ce qui est vrai, par contre, c'est que personne n'aime accomplir des tâches inutiles ou imposées, ou travailler pour le bénéfice de purs étrangers.

Il n'est pas vrai non plus que l'homme soit un être naturellement compétitif plutôt que coopératif (vii.). Nous sommes tous doués pour la coopération aussi bien que pour la compétition et la plupart d'entre nous davantage pour la première que pour la seconde. Autrement, nous serions inaptes à faire partie utilement des systèmes sociaux, qu'il s'agisse de la famille ou des organisations internationales. Exagérer l'importance de la compétition aux dépens de la coopération -ce qu'ont fait les philo-

D.O. Hebb, « Drives and the CNS » (conceptual nervous system), Psychological Review, no. 62, 1955. 
sophes dialectiques, les darwinistes sociaux, Freud, Konrad Lorenz et les économistes, tant classiques que néo-classiques - empêche de concevoir l'existence même de systèmes sociaux ${ }^{5}$.

La loi (viii.) de l'utilité marginale décroissante est intuitivement juste. Toutefois, 1) elle entre en contradiction avec le postulat (ii.) de l'insatiabilité ; 2) elle est difficile à vérifier car, par définition, l'utilité (valeur subjective) n'est pas objective et n'est donc pas facilement mesurable ; 3) il serait souhaitable de faire découler cette loi de celles qui se rapportent aux besoins objectifs.

Quant à (ix.), s'il est vrai qu'il faille opérer des choix et prendre des décisions à chaque étape, ce n'est jamais en toute liberté que nous le faisons. Nous sommes soumis à des contraintes diverses, certaines biologiques, d'autres sociales, en particulier, ceux d'entre nous qui ne sommes ni dirigeants ni entrepreneurs mais esclaves, serfs, ménagères, prisonniers, travailleurs non-syndiqués, chômeurs, marginaux, soldats ou encore professeurs, prêtres, soit tous ceux qui ne sont pas en mesure de prendre de décisions d'ordre économique qui aient une portée quelconque. La théorie tout entière du choix social, avec ses amusants paradoxes et les solutions ou fausses solutions ingénieuses qu'on a pu lui apporter, peut à la rigueur s'appliquer à des problèmes banals qui ne requièrent aucune théorie. Mais, cette approche ne nous est d'aucun secours lorsque les choix ont déjà été faits pour nous, sans parler des questions de vie ou de mort. Dans de telles situations, qui sont probablement les plus courantes, nous faisons tous face au dilemme de Portia : «ô, que faire, ce mot de 'choix' ! (...) Je suis capable ni d'en choisir un, ni d'en refuser aucun ». (Le Marchand de Venise, I, ii).

La dernière hypothèse, selon laquelle l'homme rechercherait l'optimisation (x.), est peut-être la plus typique de tous les postulats psychologiques de l'économique classique et néo-classique. Cela paraît si évident que Samuelson déclarait : "il ne s'agit pas simplement d'une loi de l'économique, mais bien d'une loi de la logique » ${ }^{6}$. Une affirmation que tout logicien récuserait. Ce postulat est également l'un des plus anciens ; en fait, il remonte à près de deux siècles, mais ce n'est que récemment qu'on l'a remis en question, pour des raisons méthodologiques et empiriques. D'abord, l'hypothèse est ambiguë : le profit doit-il être maximisé à court, moyen ou long terme ? Ce n'est pas là une question gratuite : en effet, nous sommes tous disposés à sacrifier des avantages à court terme, si besoin est, pour nous assurer des bénéfices à long terme, ou inversement. D'ailleurs, cette hypothèse n'a pas très bien résisté aux épreuves empiriques. Premièrement, la plupart des gens ne se comportent pas rationnellement dans des situations de choix, en particulier, ils n'agissent pas de façon

M. Bunge, A World of Systems, Boston-Dordrecht, Reidel, 1979.

P.A. Samuelson, Economics, New York, McGraw-Hill, 10e édition, 1976. 
à optimiser les biens et services souhaités et même, très souvent, ne savent pas cerner correctement les possibilités de choix ${ }^{7}$. En second lieu, les gestionnaires semblent généralement préférer la croissance à la rentabilité à long terme ${ }^{8}$. Et, naturellement, en période de récession, les administrateurs prudents en sont réduits à ne rechercher que la simple survie. Ces faits constituent une réfutation sans réplique de la psychoéconomique néo-classique et, par conséquent, de la théorie de l'entreprise qui s'appuie sur elle. Pour cette raison, Simon a proposé une alternative, soit le concept de satisfaction ${ }^{9}$. Plutôt que d'attendre que toutes les solutions de rechange aient été réunies et évaluées, le décideur essaie de les découvrir et son travail s'interrompt dès qu'il croit en avoir saisi une qui ne se répétera peut-être pas. Cela en fait donc non pas un optimisateur, mais quelqu'un qui recherche la satisfaction. Bien que cette alternative à l'optimisation présente des problèmes, elle semble préférable à la doctrine classique ${ }^{10}$.

Ayant suffisamment commenté les présupposés de l'économique néo-classique, nous nous tournons maintenant vers l'analyse des postulats explicitement formulés par ces théories. Alors que certains d'entre eux sont vérifiables de façon immédiate, d'autres doivent être étayés de présuppositions et de données. Considérons d'abord les premiers et, plus particulièrement, les postulats suivants de l'économique classique et néo-classique :

A. L'économie de marché constitue un système auto-régulateur, c'est-à-dire qu'il est toujours équilibré et qu'il passe constamment d'une position d'équilibre à une autre.

B . Dans l'économie de marché, les prix ne sont fixés ni par les producteurs, ni par les consommateurs, ni par personne d'autre : ils sont le résultat du libre jeu de l'offre et de la demande.

L'objection la plus patente que soulèvent ces postulats est que leur présupposé renvoie à une situation révolue. En fait, il n'existe plus, dans les pays industrialisés, de marchés libres ou concurrentiels, du moins au niveau du grand commerce et de la grande industrie qui relèvent principalement d'immenses sociétés, qu'elles soient privées ou gouvernementales, régies par tout un dispositif légal et bureaucratique, et

D. Kahneman et A. Tversky, « On the psychology of prediction », Psychological Review, no. 80, 1973, pp. 237-251. A. Tversky et D. Kahneman, « The framing of decisions and the psychology of choice », Science, no. 211, 1981, pp. 453-458.

8 J.R. Hiller, «Long run profits maximisation : an empirical test », Kyklos, no. 31, 1970, pp. 475490.

9 H.A. Simon, «A Behavioral Model of National Choice », Quarterly Journal of Economics, no. 69, 1975, pp. 99-118.

10 M. Bunge, Economia y filosofia, Madrid, Technos, 1982. 
tenues en respect par de puissantes (et donc facteurs d'affaiblissement) organisations syndicales.

Le trait marquant de l'économie capitaliste contemporaine n'est pas la libre entreprise (ou polypole) mais bien la concentration (ou oligopole). Ainsi, aux États-Unis, les équipements de transport, les métaux primaires, les produits chimiques, les machines électriques et d'autres marchandises pondéreuses sont tous entre les mains de quatre sociétés au plus ${ }^{11}$. Pour ce qui est du Tiers-Monde, des sociétés transnationales détiennent un monopsome (monopole d'achat) sur les cultures d'exportation, tandis que le plus grand nombre des industries employant des technologies avancées sont la propriété de sociétés transnationales qui doivent leur remettre des redevances substantielles en échange de l'exploitation de leurs brevets. La concentration du capital n'est pas non plus sans précédent historique : les historiens de l'économique observent que le marché a toujours, même pendant le 19e siècle, été altéré par les monopoles et contraint par des lois. D'où l'hypothèse que le marché est une entité autorégulatrice «contient un élément de vérité, un élément de mauvaise foi et, aussi, un élément d'aveuglement délibéré » ${ }^{12}$. Et pourtant, le savant Journal of Economic Theory publiait, en 1980, les actes d'un colloque consacré à la théorie de la concurrence parfaite. En 1983, le prix Nobel d'économique était décerné à Debreu pour avoir démontré que l'équilibre général, c'est-à-dire l'équilibre de tous les marchés, était possible dans une économie concurrentielle.

Une deuxième objection serait que, puisque la libre concurrence n'existe pas sauf dans les manuels et revues d'économique, le mécanisme qui était censé assurer l'équilibre a également disparu : l'économie moderne n'est pas un système autorégulé géant qu'il vaudrait mieux laisser fonctionner sans intervention. La Grande Crise et les diverses récessions qui ont suivi ont démontré qu'il n'y a tout simplement pas de chose telle qu'un équilibre permanent, et surtout universel. Il n'y a donc pas de «main invisible » (Adam Smith) capable de stabiliser l'économie. Il existe plutôt, de façon très manifeste, les pattes de la Grande Entreprise, des Grands Gouvernements et des Grands Syndicats.

Comme on le sait, les politiques fiscales keynésiennes ont été mises en oeuvre au début des années 1930 parce que les économies capitalistes couraient alors au déséquilibre. Et partout où l'on prend de ces mesures de stabilisation, l'amplitude des fluctuations commerciales est contenue ; malgré que, parallèlement, surgissent de nouveaux conflits. En effet, les objectifs des autorités étatiques (planificateurs, banques centrales, ministères des finances, organismes de conservations, agences de

J.M. Blair, Economic Concentration, New York, Harcourt-BraceJovanovich, 1972.

F. Braudel, Afterthoughts on Material Civilizationand Capitalism, Baltimore, Johns Hopkins University Press, 1977, p. 44. 
développement, etc.) peuvent ne coïncider nullement avec ceux des gestionnaires, qu'ils soient capitalistes ou socialistes.

Pour nous résumer, l'économie de marché, qui est le sujet même des théories économiques classique et néo-classique, a disparu, de sorte que ces théories ne présentent plus qu'un intérêt historique. Quant aux tentatives de ressusciter ce type d'économie par le simple expédient de lever les contraintes gouvernementales, elles sont vouées à l'échec, à moins d'être imposées impitoyablement, à la façon des dictatures fascistes. Premièrement, parce que ces mesures ne touchent pas les grandes sociétés qui comme l'avaient bien vu Adam Smith et Karl Marx - réduisent substantiellement l'essence de la libre entreprise, c'est-à-dire la concurrence. Deuxièmement, parce que ces lois, tant honnies par les profiteurs d'une économie de marché, constituent la seule protection de la petite entreprise et du public. En conséquence, l'élimination de l'Étatprovidence au nom d'une économie d'aide sociale ne peut que mener à la misère, au mécontentement et, par suite, à la révolte.

Si l'on revient au postulat B, selon lequel le prix d'équilibre est fixé par le libre jeu de l'offre et de la demande, il a connu le même sort que le postulat A. Il ne vaut pas, là où certains secteurs de production - dans certains cas l'industrie, dans d'autres, l'agriculture - sont protégés ou encore subventionnés, alors que d'autres créent des demandes au lieu d'y répondre. Il ne vaut pas non plus, là où les moyens de production sont entre les mains de monopoles ou d'oligopoles qui préfèrent maintenir des prix élevés plutôt que de vendre à moindre profit. C'est ainsi que lorsque la demande chute, c'est plutôt les prix, la production et la main-d'œuvre qui subissent des coupures. Ce phénomène, la course aux armements et les attentes des producteurs et des consommateurs, qui accomplissent leurs propres prophéties quant aux hausses de prix, sont les causes principales de la stagflation qui sévit dans les pays membres de l'OCDE. Dans les pays en voie de développement, une rapide croissance démographique à laquelle ne répond pas une capacité de production suffisante, jointe à une corruption endémique et aux énormes investissements dans les armements et dans la bureaucratie, produit des effets analogues. Bref, au contraire de ce qu'affirme la théorie du consommateur, de nos jours, les prix ne sont pas déterminés par le jeu de l'offre et de la demande. Enfin, ce postulat ne vaut pas, là où des forces économiques autres que celles du commerce et du public, comme les syndicats et les gouvernements, font sentir leur poids.

Si l'on conserve encore des doutes sur la fausseté du postulat B, il suffit de jeter un coup d'œil aux données. On y voit clairement que ce sont les grandes sociétés qui fixent les prix ${ }^{13}$. On y suit habituellement la méthode du prix de revient prévu,

J.K. Galbraith, Economics and the Public Purpose, Boston, Houghton-Mifflin, 1973. 
majoré d'un pourcentage fixe, sans égard à la demande ${ }^{14}$. Il s'agit là, on s'en souviendra, du même procédé qu'utilisa d'abord l'OPEP pour fixer les prix du pétrole brut. De sorte que personne, à l'exception de certains étudiants et professeurs d'économie, ne semble croire à l'utilité des fonctions de la demande (celles à courbe négative, rappelons-le) dans le calcul des prix. On ne possède encore aucune théorie réaliste de formation des prix. Tout ce que nous savons au sujet de ceux-ci, c'est qu'ils ne sont pas fixés comme le pensaient les économistes d'obédience classique et néo-classique.

La conclusion est limpide : les postulats de base des théories économiques classique et néo-classique, en particulier de celles des écoles de Walras et de Marshall, sont, dans le meilleur des cas, impossibles à vérifier et, dans le pire, dénuées de substance. Ce ne sont plus que des curiosités historiques. Pis encore, comme Lord Kaldor l'écrivait, «le puissant attrait des habitudes de pensée engendrées par l'économique de l'équilibre est devenu un obstacle majeur au développement d'une économique scientifique ${ }^{15}$.

Voilà pour ce qui en est des fondements théoriques de l'économique du néo-conservatisme. Penchons-nous maintenant sur les politiques prônées et appliquées en vertu de ce fatras idéologique.

\section{La politique économique}

$\underline{\text { Retour à la table des matières }}$

Quittons les abstractions de l'économique positive pour passer aux réalités de l'économique normative ou politique. Cette dernière, comme tout autre domaine de connaissance, possède une problématique qui lui est propre. Les problèmes que les économistes politiques doivent tenter de régler sont des problèmes de macroéconomie, ancrés dans la vie de tous les jours, tels que les taxes et la stagflation. Cela peut paraître évident mais est loin de l'être dans la mesure où il n'y a pas de problèmes purement économiques. Ce que nous désignons habituellement sous le terme «problèmes économiques » sont de fait des «problèmes sociaux » à multiples

Silberston 1970 .

N. Kaldor, «The Irrelevence of Equilibrium Economics », Economics Journal, no. 82, 1972, p. 1237. Pour d'autres critiques, se reporter à Lange 1963, Diamand 1973, Galbraith 1973, Perroux 1975, Hollis et Nell 1975, Katouzian 1980, et Thurow 1983). 
facettes où jouent non seulement l'économie, mais encore la politique et la culture. Cela, pour la simple raison que chacun de ces derniers domaines constitue un soussystème de la société ${ }^{16}$.

Par exemple, on a identifié quatre facteurs à la source de l'inflation mondiale amorcée dans les années $1970{ }^{17}: 1$ ) la croissance sans précédent de la production et de la consommation de biens ; 2) la révolution qui a eu lieu en publicité, qui ne cesse de créer de nouveaux besoins ; 3) la révolution keynésienne qui a permis d'éviter les récessions cycliques, lesquelles antérieurement interrompaient ou renversaient le processus de croissance et (4) l'explosion des dépenses d'ordre militaire qui atteignent actuellement près de deux mille millions de dollars par jour. Ces facteurs sont tous des variables non monétaires. Il n'est donc pas mystérieux de constater que la théorie du monétarisme soit impuissante à expliquer le phénomène de l'inflation et, à plus forte raison, celui de la stagflation ${ }^{18}$.

Nous ne voulons aucunement nier la possibilité que l'économique devienne un jour une science digne de ce nom. On peut arriver à des modèles purement (ou presque) économiques, tel que le serait un modèle conçu pour l'exploitation d'une mine de cuivre, ou encore pour la commercialisation d'un type de biens. Même si, pour être réalistes, de tels modèles devront intégrer certaines variables autres qu'économique : physiques, biologiques, psychologiques et politiques. Ce que nous voulons dire ici, c'est qu'il ne peut y avoir de politiques et de plans efficaces qui soient de nature exclusivement économique qui ignoreraient donc les phénomènes non-économiques. Et c'est là justement l'une des graves lacunes des politiques économiques néo-conservatrices; elles ne tiennent aucunement compte du fait que l'économie, loin d'être un système isolé, autonome, n'est qu'un sous-système de la société et, en conséquence, précisément tout le contraire d'autonome et donc d'autorégulé.

La raison qui explique l'inefficacité des politiques exclusivement économiques est la suivante. Les politiques doivent, en principe, être appliquées, à leur avantage ou à leur désavantage, par des êtres humains et non par des robots. Il s'avère que les être humains sont non seulement des agents ou des victimes sur le plan économique, mais également sur les plans culturel et politique. On admet facilement que des désastres naturels ou sociaux, tels un tremblement de terre dévastateur, une grève qui paralyse la société ou une guerre, peuvent retarder l'application d'un modèle de production ou de commercialisation, où que ce soit. Mais le même modèle, avec des modifications possibles, peut être mis en oeuvre une fois que la société s'est relevée des effets de la

M. Bunge, A World of Systems, op. cit.

R. Triffin, «The Future of the International Monetary System», Banka Nazionale del Lavoro Quarterley Review, no. 132, 1980, pp. 29-55.

18 M. Bunge, «La crisis actual no data de ayer ni es exclusivamente economica », Boletin de Estudios Economicos, (Bilbao) no. 38, 1983, pp. 43-45. 
catastrophe. De plus, il pourrait peut-être servir dans des économies capitalistes, socialistes ou mixtes. D'autre part, une politique économique peut échouer à cause d'un manque de coopération du publie, ou encore devenir périmée du jour au lendemain à la suite de bouleversements sociaux. Les politiques économiques sont des politiques sociales et non des théories scientifiques ; elles ne sont pas là pour décrire la situation existante, mais bien pour proposer des mesures à prendre.

Pour la même raison, les économistes ne sont pas mieux placés pour préconiser des politiques sociales que les sociologues, les politicologues, les éducateurs ou tout autre spécialiste d'un domaine des sciences ou des technologies sociales. En vérité, toute politique sociale, même si elle déclare ne viser qu'un secteur de la société, agira vraisemblablement sur tous les autres. Par conséquent, si la politique est conçue par des spécialistes d'un seul domaine, il est probable qu'elle sera préjudiciable à tous les autres. La leçon est facile à tirer : toute politique sociale adéquate du point de vue technique (et moral) devrait être élaborée par une équipe pluridisciplinaire.

Nous venons d'introduire la notion de moralité qui, absente de l'économique positive, est cruciale pour les politiques économiques ${ }^{19}$. L'élaboration d'une politique économique n'est vraiment pas une question uniquement de technique économique comparable, disons, au fait de dresser les budgets d'une firme ou de concevoir une campagne commerciale. La conception des politiques économiques appelle, ou le devrait, un modèle de l'ensemble de la société, de même que des critères de valeurs et d'objectifs.

Cette formule est amplement démontrée par le contraste que l'on constate entre les politiques keynésienne et monétariste. Tant Keynes que Friedman se sont portés à la défense du capitalisme mais, alors que le premier désirait l'humaniser, le second semble avoir voulu lui redonner la férocité qui le caractérisait pendant l'ère victorienne. Keynes était motivé non seulement par une vue moins unilatérale de la société mais aussi, peut-être primordialement, par son horreur de la guerre et sa compassion pour les chômeurs ${ }^{20}$. On ne retrouve pas cette horreur de la guerre ou cette compassion pour l'être humain dans les écrits du Professeur Friedman ou dans ceux de ses collègues de l'école de Chicago qui prônent un « taux naturel et normal de chômage » pour maintenir à la baisse les salaires et, par suite, les prix ${ }^{21}$.

On connaît, bien sûr, plusieurs types de politiques économiques, mais elles ont toutes en commun certains traits méthodologiques que nous allons tenter de faire ressortir. Les principaux types en sont le libéralisme, l'interventionnisme et la planifi-

Voir G. Myrdal pour un exposé de l'importance des valeurs en recherche sociale appliquée.

Klein, 1947.

M. Friedman, «The Role of Monetary Policy », American Economic Review, no. 58, 1968, pp. 117. 
cation centralisée. Le premier englobe en fait une non politique, car il recommande le laisser-faire. Son fondement théorique réside dans le mythe selon lequel l'économie est une machine ou un organisme doté de mécanismes auto-régulateurs. Il est évident que l'actuel système a des points faibles comme des points forts, mais on ne peut rien à cela. Les mesures gouvernementales peuvent être soit inefficaces, soit efficaces ; mais, en ce dernier cas, elles ont des effets négatifs, car elles empiètent sur la «liberté », comprise dans le sens de la libre entreprise et non, il va sans dire, dans celui de la libération de la pauvreté. La caution morale, ici, repose sur l'idée suivante : la valeur suprême est la liberté de l'individu, elle comporte le droit de disposer librement de ses avoirs ${ }^{22}$.

Le deuxième type de politique, l'interventionnisme, connaît diverses tendances, depuis les politiques fiscales générales visant à stabiliser l'économie jusqu'aux politiques à la scandinave se proposant de diminuer les inégalités par une redistribution des ressources. Fondement théorique : l'économie n'est pas naturellement en équilibre, comme nous le rappelle chaque récession; il faut donc prendre les moyens de l'aiguiller vers des États d'équilibre «relatif ». Caution morale : les valeurs primordiales sont représentées par le bien de l'individu ainsi que par l'homogénéité et la stabilité de la société. Puisque ces valeurs sont incompatibles avec les creux des cycles financiers, l'économie doit faire l'objet de dirigisme. Le monétarisme est libéral en théorie, mais interventionniste en pratique, car il aspire à la réinstauration de l'économie de marché, ce qui nécessite l'élimination de l'État-providence, au risque même de rébellions ou au prix d'une dictature. Le discours monétariste, on le voit, est contradictoire.

Le troisième type de politique, soit la planification centralisée, sert non seulement à la régulation des économies socialistes, mais entre aussi en jeu dans les économies mixtes de certains pays dont le Royaume-Uni, la Norvège et les Pays-Bas. Dans le Tiers-Monde, on l'utilise comme guide du développement. Dans ce dernier cas, il trouve son fondement théorique parfois dans la théorie marxiste de l'accumulation primitive du capital, parfois tout bonnement dans la thèse qui veut que là où il n'existe pas de classe indigène d'entrepreneurs, I'État doit assumer le rôle dirigeant dans l'économie, car il est le seul agent à disposer des ressources financières et du pouvoir politique pour le faire. Fondement moral : le sous-développement économique est dégradant.

On constate donc que chacune des politiques économiques est basée sur une doctrine économique et sur un système de valeurs et de normes, malgré que cela soit rarement formulé de façon explicite. Par ailleurs, un plan économique spécifique

F.A. Hayek, Individualism and Economic Order, London, Routledge and Kegan Paul, 1949, The Counter-Revolution fo Science, Glencoe, Free Press, 1955. 
repose sur : 1) une politique spécifique, 2) un quelconque modèle, soit d'un secteur économique, soit de l'économie tout entière, soit mieux encore, de l'ensemble de la société, 3) des données plus poussées. Schématisons :

$$
\begin{aligned}
& \begin{array}{l}
\text { POLITIQUE } \\
\text { PLAN }
\end{array} \\
& \text { THÉORIE + DONNÉES + SYSTÈME DE VALEURS, } \\
& \text { P'où } \\
& \text { PLAN }
\end{aligned}
$$

Il semble logique de stipuler qu'un plan puisse être dit scientifique si, et seulement si, il est fondé sur : 1) des théories, modèles et données scientifiques, 2) un système de valeurs et de normes compatible avec l'acquis scientifique actuel. L'absence de l'un ou l'autre de ces composantes le condamne à l'échec, à des dépenses sociales considérables. Notons, en particulier, qu'une théorie économique fausse ou un code moral périmé - impossible à appliquer - empêchera la réussite du plan économique le plus soigneusement élaboré.

Un individualisme extrême, que ce soit en théorie économique ou en philosophie morale, présente une bonne illustration du phénomène. Il ne peut réussir à long terme parce que la société - dont l'économie n'est qu'un des sous-systèmes - est justement un système et que la durée de tout système exige un certain degré de coopération, volontaire ou non. Il est vrai que l'individualisme n'exclut pas la solidarité, mais restreint cette dernière aux membres d'une même couche sociale : elle est horizontale et défensive, et non pas intégrale et altruiste. De plus, les systèmes artificiels dont l'économie, la culture et le régime politique, appellent, par leur nature même, des mécanismes de contrôle ou de régulation. Il n'y a pas de machine qui puisse fonctionner sans service d'entretien et de réparation.

Un autre bon exemple de ce qu'est une mauvaise politique sociale est l'économisme, soit la croyance que l'économie est, ou la grande force motrice de la société, ou encore qu'il est possible de l'isoler des autres sous-systèmes qui en font partie intégrante : biologie, culture et régime politique. Faux modèle de la société, l'économisme est la source de politiques économiques, tant capitalistes que socialistes, qui ont fait un tort incalculable à la société tout entière, qu'elles échouent ou qu'elles réussissent à stimuler, temporairement, l'économie. Puisque l'économique ne peut refléter qu'une facette du tissu social, les plans et politiques économiques devraient faire partie d'un cadre général de plans et de politiques sociales. La théorie économique divorcée de la réalité est une pure fiction, mais une politique économique reposant sur 
une théorie divorcée de la réalité est, dans le meilleur des cas, inefficace et, au pire, criminelle.

Fait paradoxal, les économistes néo-conservateurs et marxistes adoptent, les uns comme les autres, le dogme de l'économisme. La version la plus poussée de ce dogme est le monétarisme, cette politique selon laquelle tout ce qu'il faut faire pour maintenir l'économie en bonne santé - ou du moins pour garantir le bonheur des capitalistes - est de surveiller la masse monétaire ${ }^{23}$. Immanquablement, le monétarisme évoque les panacées de jadis : infusions de goudron, manipulations des vertèbres et saignées, cette dernière surtout, en l'occurrence. Tout comme la sorcellerie, on l'adopte sur parole plutôt que sur la foi de théories et de statistiques. On y adhère pour deux raisons : à cause de sa simplicité, aussi bien théorique que pratique, et parce qu'elle privilégie les riches. Sa simplicité théorique est évidente : dans l'économique vulgaire, l'argent est l'alpha et l'oméga. Sa simplicité pratique parle également d'elle-même : de toutes les variables économiques, la masse monétaire est celle qui se prête le mieux à la régulation. On comprend sans difficulté l'attrait du monétarisme pour les conservateurs ; il prône en effet l'abolition de l'État-providence et le dégagement des entrepreneurs de toute obligation envers la société. La seule responsabilité d'ordre social des administrateurs de sociétés est « de faire en sorte que leurs actionnaires touchent des gains aussi élevés que possible ${ }^{24}$.

Qu'on n'adopte pas nécessairement le monétarisme à cause de sa cohérence théorique ou parce que les statistiques sont venues confirmer son efficacité ne veut pas dire qu'il manque de tout fondement théorique ou statistique. Il intègre ces deux composantes et aussi une méthodologie propre et, comme nous le disions ci-dessus, la morale du darwinisme social la loi de la jungle, ni plus ni moins. La théorie de base du monétarisme est celle que proposait Adam Smith en 1776 : une économie de marché exempte des tares que lui a infligées le 20e siècle, dont les monopoles, les oligopoles, des gouvernements ultra-puissants et des syndicats également ultra-puissants. Friedman a aussi conservé l'axiome néo-classique selon lequel les gens d'affaires « rationnels » agissent de façon à optimiser les profits et, pour ce faire, se procurent et exploitent toutes les données et ont la prévoyance nécessaire. Cette règle n'étant pas forcément probante, il y ajoute une clause curieuse. Il nous dit qu'il est égal que les prémisses néo-classiques soient justes ou non, l'important étant que « les firmes particulières se comportent comme si elles cherchaient rationnellement à optimiser les bénéfices prévus et avaient pleine connaissance des données nécessaires pour atteindre leur but ${ }^{25}$. En conséquence, il se sent dégagé de l'obligation de mettre ces

\footnotetext{
M. Friedman, Inflation, Causes and Consequences, New York, Asia Publishing House, 1963, The role of monetary policy, op. cit., Free to Choose, New York, Avon Books, 1980.

M. Friedman, Inflation ... op. cit., p. 133.

M. Friedman, «A Theoritical Framework for Monetary Analysis », RJ. Gordon, ed. Milton an's Monetary Framework, Chicago, University of Chicago Press, 1970.
} 
prémisses à l'épreuve pour découvrir si elles sont justes : qu'elles le soient ou non, tout se passe comme si elles l'étaient, on se croirait au Pays d'Oz. Ce trucage méthodologique, glané dans la philosophie fictioniste des Nietzsche et des Vaihinger, est si bien connu qu'il a un surnom, le F-Twist ${ }^{26}$. Notons le gouffre qui sépare le monétarisme de la science qui, elle, colle aux faits et non à la fiction ${ }^{27}$.

Mais l'apport le plus important du Professeur Friedman à l'armature conceptuelle des politiques monétaristes est son « cadre théorique d'analyse monétaire ${ }^{28}$. Il a tout à fait raison dans son utilisation de «cadre théorique », et il serait encore plus près de la vérité si sa formule se lisait «billet d'ordre théorique », car il ne s'agit pas d'une théorie a proprement parler. En fait, ce « cadre » renferme trois symboles de fonctions nullement définies ( $\mathrm{f}$, g et 1 ), si bien que les formules où ils apparaissent sont des formules ouvertes et vagues du type « $\mathrm{Y}$ est une fonction (inconnue) de $\mathrm{X} »$. La politique économique tout entière de Friedman se fonde ainsi sur un programme de théorie et non sur une théorie précise. Étant un programme (à être mis en oeuvre par quelqu'un), cette politique est incapable d'expliquer, et encore moins de prévoir, quoi que ce soit. Et, puisque la prévision lui est impossible, elle ne peut être soumise à l'épreuve. Impossible à mettre à l'épreuve, elle n'est donc même pas fausse. Cette lacune fatale ne troublerait peut-être pas un écrivain imaginatif mais, ici, c'est le signe qu'on a affaire à une pseudo-science.

Les monétaristes, donc, ne possèdent pas de théorie de l'inflation et, par la suite, ne disposent d'aucun argument sérieux qui justifierait leurs solutions pour résoudre le problème de l'inflation. Mais, bien sûr, ils ont formulé une hypothèse quant à la source de l'inflation, et dérivé une règle de cette hypothèse. Cette hypothèse, simpliste, suggère que l'inflation a une seule cause, c'est l'augmentation excessive des signes monétaires ; le remède est donc d'arrêter les émissions. L'hypothèse est trop simple pour être juste. Ce serait plutôt le contraire qui le serait, c'est-à-dire que le rythme avec lequel un gouvernement injecte de la monnaie dans le circuit est déterminé par le déséquilibre entre les dépenses et les revenus. Si les premiers excèdent les seconds, le gouvernement émet ou emprunte de la monnaie afin de répondre à ses obligations ; seul un budget strictement équilibré peut en arriver à un taux zéro d'augmentation de la monnaie. En outre, dans un État-providence, les dépenses sont en fonction linéaire croissante avec le chômage, les chômeurs ne consommant que le minimum et ne payant guère d'impôts. La conclusion de tout cela, c'est que le rythme auquel le gouvernement imprime ou emprunte de la monnaie dépend linéairement du taux de

26 P.A. Samuelson, «Contributions to the Discussion on the Problems of Methodology », Proceedings of the American Economic Association, no. 59, 1963, pp. 229-231.

M. Bunge, Understanding the World, op. cit.

M. Friedman, Milton Friedman's Monetary Framework, op. cit. 
chômage. Bref, le chômage cause l'inflation qui, à son tour, augmente le taux de chômage. ${ }^{29}$.

En ce qui touche au support statistique du monétarisme, il se résume à certaines corrélations discutables, interprétées comme des relations causales. Pour Lucas par exemple, les données existantes sur l'économie américaine pour la période de 1953 à 1977 confirment les hypothèses qu'un taux de changement donné dans la quantité de monnaie entraîne 1) un changement correspondant dans le taux d'inflation des prix, et 2) un changement correspondant dans les taux d'intérêt nominal. En fait, tout ce que ces données démontrent est une corrélation possible, et non une relation de cause : le mécanisme sous-jacent n'a pas été étudié ${ }^{30}$. De plus, ces preuves sont douteuses. Deux exemples suffiront à l'illustrer.

Modigliani, lauréat du prix Nobel en 1985, a examiné approximativement la même tranche chronologique de l'économie américaine, en se concentrant sur les périodes où la masse monétaire connaissait une croissance relativement continue. Il a cerné deux de ces périodes : du début de 1953 à la première moitié de 1957, et de 1971 à $1975^{31}$. Or, ces périodes se sont révélées être les plus instables de toutes, si l'on tient compte du recul de 1954, de la reprise de 1955, de la récession de 1958 et de celle de 1973-1975. La conclusion qui s'impose est la suivante : « Nos périodes les plus instables ont été des périodes de stabilité monétaire relative ${ }^{32}$. Des statistiques plus récentes viennent étayer cette constatation. La récession économique qui débuta aux États-Unis vers 1978 a d'abord été accompagnée de restrictions, puis d'une réduction des réserves monétaires $(\mathrm{MI})$; la réduction a été encore plus grave au Canada, où la crise était plus aiguë. Les changements de pourcentage aux États-Unis entre 1976 et 1982 ont été de 1,0 2,3 0,8 0,9,-1,6 et 3,8, respectivement ${ }^{33}$. À ce moment, l'échec de la politique monétaire était devenu si flagrant que l'administration Reagan abandonna, à toutes fins pratiques, cette dernière, ce qui a permis la reprise partielle de 1983.

Cependant, le camouflet idéologique le plus retentissant que reçut le monétarisme lui fut infligé par une équipe d'universitaires, conseillers auprès de la Bank of England, cette forteresse du monétarisme ${ }^{34}$. Dans leurs rapports, les Professeurs A.J. Brown, D.F. Hendry et N. Ericsson mettaient en pièces l'ouvrage de Friedman et

Pour un exposé mathématique voir M. Bunge, Understanding the world, op. cit.

R.E. Lucas, «Two Illustrations of the Quantity Theory of Money », American Economic Review, no. 70,1980 , pp. 1005-1014.

31 F. Modigliani, «the Monetarist Controversy, or, should we Forsake Stabilization Policies », American Economic Review, no. 67, 1977, pp. 1- 19.

Ibid, $p .12$.

FMI, 1992, p. 202.

Bank of England, Panel of Academic Consultants, Paper no. 22, London. 
Schwartz ${ }^{35}$, où ceux-ci déclaraient avoir prouvé, statistiques à l'appui, que les périodes de prospérité au Royaume-Uni aussi bien qu'aux États-Unis, entre 1867 et 1975, étaient attribuables à l'application de politiques monétaristes. Le verdict des experts fut formel : bien que les auteurs avaient déformé les données pour servir leurs fins et avaient omis d'employer certaines techniques récentes de l'économétrie, les résultats n'appuyaient nullement leurs affirmations.

En conclusion, le monétarisme est théoriquement faux et, pratiquement parlant, inefficace sinon désastreux. Pour ce qui est des philosophies politique, sociale et morale qui le sous-tendent, ce sont essentiellement celles du libéralisme économique qui ressortent, c'est-à-dire l'individualisme. Cette doctrine se réduit 1) à l'égoïsme le plus pur «Chacun pour soi »;2) au darwinisme social «Seuls les plus aptes survivent et méritent la survie »; 3) à des principes politiques libertaires, qu'on doit clairement distinguer de la notion de démocratie, soit la participation réelle et universelle de la population à l'administration de la chose publique. Notre position est que le premier principe est non seulement méprisable, mais également inefficace, sauf dans la détérioration du tissu social, car la vie en société demande une mesure de coopération et d'altruisme. Je pense aussi que le darwinisme social a depuis longtemps été réfuté, si ce n'est que parce que 1) dans la vie réelle beaucoup d'entreprises bien établies font banqueroute, alors que nombre d'initiatives échevelées réussissent, et que 2) la prétendue « stricte discipline du marché » engendre non seulement des dirigeants légitimes mais aussi des escrocs et des oppresseurs.

Quant au lien entre la liberté politique et la liberté économique, il semble qu'il s'agisse encore une fois d'une corrélation provisoire, erronément interprétée comme une relation causale permanente. En réalité, même si le laisser-faire économique a été relié aux libertés civiles dans quatre pays (la France, la Grande-Bretagne, les PaysBas et les États-Unis), et y a certainement favorisé l'expansion du capitalisme, ce lien ne tient plus nulle part aujourd'hui, car les oligopoles et l'intervention étatique ont étouffé la libre entreprise. En outre, maintenir avec succès une libre entreprise sans contraintes aucunes demanderait un gouvernement autoritaire, disposé à réprimer impitoyablement toute menace à la liberté économique, notamment celles que constituent les syndicats, les groupes politiques favorisant la nationalisation des services publics et des ressources énergétiques, et même le mouvement coopératif. Le néolibéralisme est ainsi devenu l'un des grands ennemis de la démocratie ${ }^{36}$.

Chez les nations industrialisées, les politiques monétaristes entraînent l'élimination des petites et moyennes entreprises et réclament l'abolition de l'État-providence,

35 M. Friedman et AJ. Schwartz, Monetary Trends in the United States and the United Kingdom, Chicago, University of Chicago Press, 1982.

36 R. Prebisch, « Dialogo acerca de Friedmany Hayek », Revista de la CEPAL, no. 15, 1981, pp. 161192. 
ce qui accentue la pauvreté, mène au mécontentement et ouvre la porte aux répressions. Dans les pays du Tiers Monde, la répression rend possible l'application de politiques monétaristes, avec le résultat d'une misère accrue engendrant la rébellion et des répressions de plus en plus dures. C'est la raison pour laquelle le FMI, qui préconise habituellement de telles politiques dans les pays en voie de développement a été surnommé Instant Massive Fury : Soulèvements Massifs Instantanés. Il ne faut pas s'étonner que, dans ces conditions, le libéralisme économique, le monétarisme en particulier, soit devenu partout une idéologie de droite et même une grave menace pour la civilisation. Un caricaturiste mexicain résume bien la situation dans l'anecdote suivante. Dans un musée, un guide fait son boniment : «Ce tableau est de l'école de Giotto, celui-là de l'école néo-classique et l'autre, là-bas, un bon exemple de l'école impressionniste ». Puis, se retournant vers la fenêtre et pointant dans la direction de Friedmanville, un bidonville, que l'on y aperçoit, il poursuit : " Quant à celui-là, il est typique de l'école de Chicago ».

Pour nous résumer, toute politique économique a pour fondement une théorie, un corpus de données ainsi qu'un système de valeurs et de normes. Et, tout plan économique repose sur une politique économique adjointe d'un modèle économique et d'une autre banque de données. Les politiques et les plans sont scientifiques dans la mesure où leurs composantes le sont ou, du moins, sont compatibles avec les connaissances scientifiques contemporaines. Autrement, ils ne sont pas scientifiques et, donc, peu susceptibles de réussir. Le monétarisme est un cas exemplaire de politique économique non scientifique, car il repose sur 1) un modèle de la société purement économique, mono-dimensionnel, 2) une théorie économique périmée et imprécise, regorgeant de postulats non vérifiés ou même faux, 3) des analyses statistiques qui, dans le meilleur des cas, aboutissent à des corrélations, et 4) des systèmes de valeurs périmés et des principes moraux inhumains qui, s'ils étaient appliqués, ne pourraient que détruire le tissu social. En conséquence, on a toutes les raisons, scientifiques et morales, culturelles et politiques, de combattre le monétarisme. Les mêmes arguments valent, à peu de choses près, pour les autres formes de l'économique néoconservatrice. Ce sont toutes des magmas d'idées confuses au plan théorique, dénuées de tout support empirique, et elles sont injustifiables aux plans moral et politique. 


\section{Conclusion}

$\underline{\text { Retour à la table des matières }}$

Nous avons trouvé sans valeur scientifique aucune les bases théoriques de l'économique néo-conservatrice, qui intègrent les mythes de l'économique néo-classique et sont dépourvues de toute théorie de l'inflation, sans parler d'une théorie de la stagflation. En fait, cette économique ne présente même pas de théorie monétaire ${ }^{37}$. Son fondement moral n'est autre que l'individualisme d'antan, qui s'oppose aux règles les plus primordiales qui régissent le comportement social civilisé, car elle prône le plus pur égoïsme et passe sous silence toute obligation, hormis celle du profit.

Les recherches empiriques n'ont pas confirmé les dogmes de l'économique néolibérale, en particulier ceux plus radical du non-interventionnisme autrichien ou ceux de l'interventionnisme monétaire à la Friedman. Il est carrément faux que la prospérité soit toujours accompagnée ou, encore moins, déclenchée par les restrictions monétaires, ou que toutes les récessions soient causées par des impressions inconsidérées de papier-monnaie. Les statistiques non plus ne viennent pas confirmer ces doctrines. Pis encore, il n'est pas vrai que l'inflation n'a toujours qu'une seule cause : les racines de l'inflation sont multiples, économiques, sociales, politiques et même psychologiques; il est donc clair que le remède n'en peut pas être purement d'ordre financier pur $^{38}$.

Les politiques économiques du néo-conservatisme ont échoué et ne peuvent manquer d'échouer de nouveau si l'on essaie de les ré-appliquer, car elles oeuvrent dans le sens d'un retour en arrière. Dans les sociétés industrialisées, cela voudrait dire l'élimination de l'État-providence et la banqueroute d'innombrables petites et moyennes entreprises. Dans le Tiers-Monde, cela aurait l'effet de détruire, le cas échéant, des systèmes de sécurité sociale encore dans l'œuf ainsi que les embryons d'entrepri-

F. Hahn, Money and Inflation, Cambridge Mass., M.I.T. Press, 1983.

M. Bunge, La crisis actual ..., op. cit. 
ses manufacturières indigènes. Et, tout cela au nom du libre-échange, qui a fait la fortune des nations industrialisées au détriment des sous-développées.

Dans les deux cas, le néo-conservatisme économique se propose d'anéantir bien des réalisations sociales estimables. D'autre part, elle ne s'attaque à aucune des sources les plus flagrantes de l'inflation : misère, insécurité, oppression politique et décalage culturel et, en particulier, l'accroissement des forces militaires et des arsenaux. L'application des politiques économiques néo-conservatrices a pour résultat la stagnation ou le déclin général de l'économie, ce qui à son tour entraîne la formation d'une génération de gens frustrés, surtout les jeunes, qui ne peuvent absolument plus se fier aux beaux discours qu'on leur tient encore sur la libre entreprise ou sur la liberté en général. Comment ces être humains pourraient-ils espérer un avenir meilleur, alors même que les politiciens néo-conservateurs au pouvoir les empêchent de vivre leur présent?

Enfin, pour terminer, l'économique néo-conservatrice est un désastre, tant intellectuellement, politiquement que moralement. Elle n'est rien d'autre que l'idéologie des riches -cupides, faussement nostalgiques et à très courte vue - qui ne se soucient que d'un type de liberté : la liberté de gérer leurs propres affaires dans leurs propres intérêts, sans égard pour l'intérêt public. Si l'on persiste dans l'application des politiques économiques néo-conservatrices, on aboutira infailliblement à l'une ou l'autre de deux situations : l'apathie qualifiée ou la rébellion sans appel qui ne constituent certainement pas des motivations favorables à l'amélioration graduelle de notre destin. Tous ceux à qui tient à cœur une démocratie intégrale, et non simplement politique, devraient lutter contre cette idéologie passéiste et oeuvrer dans le sens d'une pensée progressiste.

L'alternative à l'économique néo-conservatrice ne peut résider dans les théories vieillies de l'école keynésienne car, il faut le reconnaître, les vues de Lord Keynes étaient limitées et elles ont mal résisté au temps parce qu'il n'a pas connu la stagflation ou l'économie de guerre en période de paix. Nous ne savons pas encore quelle pourrait être une alternative valable, mais nous pouvons formuler les conditions indispensables à toute politique économique compatible avec les valeurs d'une société démocratique. Avant tout, elle doit s'appuyer sur une théorie solide, sans laquelle aucune action rationnelle n'est possible. En second lieu, elle doit intégrer un système de valeurs humaines capable de se rallier l'opinion de tous les gens honnêtes et de bonne volonté. Troisièmement, elle doit comprendre une certaine planification souple et démocratique et non rigide ou autocratique, car cela est la condition de la sauvegarde de l'individu et de nos institutions les plus nobles contre les forces aveugles de l'économie. De toute façon, nous devons aborder l'avenir avec un esprit créateur, et 
nous débarrasser des vétustés des dogmes néo-conservateurs, contradiction dans les termes si jamais il en fût.

Mario BUNGE

Département de philosophie

Université McGill

http://www.arts.mcgill.ca/programs/philo/faculty/bunge.htm

Fin de l'article. 